\title{
CONDICIONES DE PRODUCCIÓN DE LA EDUCACIÓN INCLUSIVA
}

\section{PRODUCTION CONDITIONS OF INCLUSIVE EDUCATION}

\author{
Aldo Ocampo González \\ Centro de Estudios Latinoamericanos de Educación Inclusiva (CELEI), Chile \\ aldo.ocampo.gonzalez@gmail.com \\ https://orcid.org/0000-0002-6654-8269
}

\section{RESUMEN}

Este trabajo explora las condiciones de producción de la Educación Inclusiva. Es menester señalar que, la comprensión de su fenómeno desborda las estructuras epistemológicas dominantes heredadas $-\mathrm{y}$ sus categorías-, más bien, constituye un campo de la divergencia epistémica, enfatizando en el conjunto de condiciones que crean y garantizan su conocimiento. El trabajo analiza las dificultades analítico-metodológicas

1 Chileno. Teórico y ensayista educativo. Director fundador del Centro de Estudios Latinoamericanos de Educación Inclusiva (CELEI), institución reconocida y con estatus asociativo a CLACSO. Primer centro de investigación creado en ALAC y Chile para el estudio teórico y metodológico de la Educación Inclusiva. Profesor del Máster en Creatividad, Bienestar y Salud de la Universitat de Barcelona, España, Profesor Invitado en la Maestría en Educación desde la diversidad impartido por la Univ. de Manizales, Colombia. Profesor del Programa Oficial de Doctorado en Educación, Universidad Sek, Chile. Director de Polyphonia. Revista de Educación Inclusiva, publicación científica de CELEI. Profesor de Educación General Básica, Licenciado en Educación, Post-titulado en Psicopedagogía e Inclusión (UCSH, Chile). Magíster en Educación, mención Currículo y Evaluación, Máster en Política Educativa, Máster en Lingüística Aplicada (Univ. de Jaén, España), Máster en Integración de Personas con Discapacidad (Univ. de Salamanca, España) y Doctor en Ciencias de la Educación, aprobado Sobresaliente por Unanimidad, mención "Cum Laude" por la Universidad de Granada, España. Actualmente, cursa el Programa Oficial de Doctorado en Filosofía, impartido por la Univ. de Granada, España. Es autor de 6 libros, capítulos de libros y actas científicas, así como de más de 100 artículos entre el período 2009 a 2019. Ha impartido más de 60 conferencias, conferencias magistrales y clases magistrales en casi todo Iberoamérica por invitación gracias a sus escritos y publicaciones para inaugurar congresos en España, Ecuador, México, Perú, Brasil, Colombia, Argentina y Chile, Venezuela, entre otros. En julio de 2017, tras su participación como conferencista invitado al II Encuentro de Literatura Infantil y Juvenil (EILIJ), organizado por la Universidad Nacional del Chimborazo y la Casa de la Cultura Núcleo de Chimborazo, le fue otorgado el reconocimiento de "Ciudadano llustre", por el Distrito de Riobamba, Ecuador, por su trabajo sobre Epistemología de la Educación Inclusiva y, así también, por el Municipio de El Tambo, ciudad de Huancayo en Perú, tras su participación como conferencista en el VI Seminario Internacional de Investigación, Epistemología y Educación. En 2018, la Red Iberoamericana de Pedagogía (REDIPE), le otorgó el Premio Iberoamericano a la Investigación por la creación de la Epistemología de la Educación Inclusiva, entregado en Ecuador. Cuenta con el reconocimiento otorgado por el Congreso de la República del Perú por su aporte a la cultura y a la educación en 2018. 
ligadas a la composición de su estructura teórica, devenido en la consolidación de un terreno fundamentalmente disputado por una multiplicidad de disciplinas académicas, discursos, concepciones políticas y corrientes críticas más prominentes de nuestra época. La epistemología de la Educación Inclusiva orienta su actividad hacia la creación de conceptos, herramientas metodológicas y saberes que permitan leer críticamente el presente. Una base epistemológica de naturaleza postdisciplinar, permite contrarrestar la exclusión de métodos producto de la separación de las disciplinas. Todo ello, permite reconocer que, el objeto de la Educación Inclusiva ha cambiado, no así sus métodos de aproximación, abordaje e investigación.

PALABRAS CLAVE: condiciones de producción, diaspórismo epistemológico, heterotopicalidad, estructura teórica abierta y post-disciplinariedad.

\section{CONDITIONS OF PRODUCTION OF INCLUSIVE EDUCATION}

Abstract: this work explores the production conditions of Inclusive Education. It is necessary to point out that, the understanding of its phenomenon overflows the inherited dominant epistemological structures - and their categories -, rather, it constitutes a field of epistemic divergence, emphasizing the set of conditions that create and guarantee their knowledge. The work analyzes the analytical-methodological difficulties linked to the composition of its theoretical structure, resulting in the consolidation of a fundamentally disputed terrain by a multiplicity of academic disciplines, speeches, political conceptions and most prominent critical currents of our time. The epistemology of Inclusive Education directs its activity towards the creation of concepts, methodological tools and knowledge that allow the present to be read critically. An epistemological basis of a post-disciplinary nature allows counteracting the exclusion of methods resulting from the separation of disciplines. All this allows us to recognize that, the object of Inclusive Education has changed, but not its methods of approach, approach and research.

KEYWORDS: production conditions, epistemological diaspora, heterotopicality, open theoretical structure and post-disciplinary.

\section{1.-INTRODUCCIÓN: SOBRE LA ESCASA CLARIDAD DE LA ESTRUCTURA TEÓRICA DE LA EDUCACIÓN INCLUSIVA}

En diversos trabajos he afirmado que la Educación Inclusiva constituye un campo teórico compuesto de múltiples entradas a la comprensión de su objeto, así como, una teoría sin disciplina, devenida en una estructura de conocimiento que se nutre de aportes de diversa naturaleza, recurriendo a la ecología de saberes -concebida como un sistema de reciclaje, un mecanismo de interconexión de una multiplicidad infinita de saberes- y un examen topológico -sistema analítico mediante el cual es posible graduar los niveles de proximidad y transferencia de recursos metodológicos en la confluencia de dos o más campos del conocimiento-. La expresión 'sin disciplina' alude a la cualidad viajera de la Educación Inclusiva, esto es, su capacidad para movilizarse a través de una amplia confluencia de discursos, métodos, objetos, teorías, disciplinas, campos del conocimiento e influencias, describiéndolo como un campo no-fijo, el que no puede ser delimitado en los marcos y paradigmas de ninguna disciplina. ¿Cómo llega a ocupar esta posición? Más bien, es un saber que se construye a través de las disciplinas y sus intersecciones. La expresión 'sin disciplina' se opone y trasciende las condiciones de producción propias de las epistemologías monotópicas, encapsuladas en la unicidad de razonamientos, restringiendo el diálogo, el 
encuentro y la constelación con otras tipologías de saberes y herramientas conceptuales $y$ metodológicas. En tal caso, la expresión 'sin disciplina', se convierte en un poderoso recurso de aperturación, descentrando los mecanismos de fijeza epistemológica, configura un espacio abierto al diálogo, a la relación y al encuentro de diversos saberes, interesándose por la validación de otras formas epistemológicas posibles, ensanchando su campo de racionalidad. Por todo ello, se convierte en un campo polifónico en sus sistemas de razonamientos y mecanismos de constructividad. Reconoce que el problema de delimitación de su objeto se encuentra en sus categorías empleadas. Asume un problema morfológico invisibilizado.

En este marco, la comprensión epistemológica de la Educación Inclusiva puede ser lecturada en términos de una epistemología de la heterotopicalidad, es decir, compuesta por una variedad infinita de temas, ámbitos de discusión y objetivos analítico-metodológicos. A través de esta mirada, otorga la capacidad de ampliar sus condiciones interpretativas, fortaleciendo una comprensión adecuada de la multiplicidad de elementos que configuran sus estrategias de producción. De este modo, el pensamiento epistemológico de la Educación Inclusiva es el pensamiento de la relación, del movimiento y del encuentro. Sus condiciones de producción se encuentran estrechamente vinculadas a determinados procesos sociales -dimensión extra-teórica-, con el objeto de contribuir sustancialmente a la emergencia de procesos de liberación que favorezcan a la multiplicidad infinita de diferencias. Por todo ello, es posible afirmar que, a pesar de la nebulosa analíticometodológica que permea su estructura de conocimiento, en su versión auténtica se concibe como una estructura de conocimiento de carácter abierta. ¿De qué depende esto? En mi opinión, a raíz de la contribución de campos, tales como, los Estudios de la Mujer, los Estudios Post-coloniales y Decoloniales, los
Estudios Culturales, la Filosofía de la Diferencia, Política y Analítica, los Estudios Feministas, la Corriente Interseccional, los Estudios de Género y Queer, entre otros, han sido responsables, a través de algunos de sus planteamientos, contenidos críticos y articulaciones analíticometodológicas, de la apertura de la estructura de conocimiento de lo que hemos llamado Educación Inclusiva. No es mi intención que el lector, se quedé con la idea que de antaño, este campo de conocimiento articulaba su actividad en una estructura cerrada de carácter disciplinar, más bien, intento explicitar que, a través del arribo de contribuciones, influencias, disciplinas y marcos conceptuales y políticos específicos, han contribuido a la expansión de sus horizontes teóricos -mediante la metáfora del viaje-. Razón por la cual, es posible afirmar que, esta es una estructura y un campo de conocimiento que opera en una dimensión extra-disciplinar, es decir, posee la capacidad de conectar en funcionamiento campos alejados en su actividad científica al quehacer de la educación, entrando en contacto a través de determinados objetos y tópicos analíticos.

Es menester recordar que, la base epistemológica que sustenta la naturaleza del conocimiento de la Educación Inclusiva posee un carácter postdisciplinar, esto es, orienta su actividad hacia la producción de nuevos cuerpos de saberes -trabaja en la exterioridad epistemológica (espacio de rearticulación y apertura infinita)-. Orienta su actividad hacia la consolidación de nuevos modos de lectura y abordaje de la realidad, a partir de la extracción de los aportes más significativos de la amplia multiplicidad de objetos, disciplinas, teorías, conceptos, métodos e influencias por las que este campo se moviliza. La dimensión post-disciplinar articula su actividad en la diseminación y en el diaspórismo epistemológico -características centrales de su orden de producción-, cuya actividad no se limita a la simple afirmación de sus leyes de funcionamiento basadas en la 
dispersión, sino más bien, son complementadas a través de la traducción científica, la ecología de saberes y la examinación topológica. ¿Qué diferencias y complementariedades pueden ser establecidas entre la dimensión 'extra-disciplinar' y 'post-disciplinar'? Una primera aproximación consistiría en reconocer que ambos niveles de análisis desafían el dogma metodológico y el prejuicio elitista de tipo instrumental propio de los estudios epistemológicos tradicionales al desafiar los límites disciplinarios. ¿Cuáles son los problemas que definen su capacidad intelectual, o más bien, las ponen en riesgo? Tanto la dimensión extra-disciplinar como postdisciplinar, inscriben su trabajo en lo múltiple, es decir, en la comprensión y/o conexión de una variedad infinita de recursos intelectuales y metodológicos.

La dimensión extra-disciplinar como lo postdisciplinar quedan conectadas a través de la metáfora de 'interconexión epistemológica de saberes'. La primera, orienta su actividad científica a la conexión de campos alejados en su analítica al quehacer de la Educación Inclusiva, vinculados en alguna arista a su campo y objeto de conocimiento. La dimensión extra-disciplinar resultar fértil en la determinación multiaxial de aportes, contribuciones y campos de confluencias -cada disciplina, influencia, teoría, etc., es concebida a través de un estado de singularidad-, fortalece además, la metáfora de red reticular y la zona de contacto en las que cada uno de estos elementos son albergados. El campo de conocimiento de la Educación Inclusiva en un nivel inicial puede ser descrito en términos extradisciplinares, específicamente, por su capacidad de conectar y hacer confluir campos alejados, que a su vez, poseen la capacidad enriquecer su construcción. La dimensión post-disciplinar, por su parte, se opone a las estructuras académicas institucionales de tipo convencionales. Orienta su actividad en el movimiento a través de las disciplinas, es decir, viaja no-linealmente a través de cada una de ellas, extrayendo los aportes más significativos de estas, con el objeto de fabricar un nuevo saber. No obstante, la determinación de saberes y sus políticas asociadas, enfrentan la pregunta por las 'condiciones de legibilidad'. Sobre este particular, emerge la preocupación metodológica por las condiciones de traducción.

En mi trabajo epistemológico, la traducción, se expresa en términos de creación de condiciones delegibilidadeinteligibilidad, es decir, condiciones que permitan poner en sintonía dichos aportes con la estructura de conocimiento auténtica que este enfoque inaugura. Metodológicamente, implica defabricar determinadas formas de construcción de saberes. De modo que, la condición extra-disciplinar puede lecturarse en términos de una antesala a la construcción del objeto auténtico de la Educación Inclusiva, aclarando el conjunto de enredos genealógicos que en ella tienen lugar. A través del prisma postdisciplinar es posible articular un tipo diferente de objeto, sin que este, quede atrapado en las disciplinas -psicoanálisis epistemológico-, concebido como un nuevo campo contrario a la simple y reduccionista confluencia de enfoques tradicionales. La post-disciplina constituye una estrategia de dislocación y reinvención crítica ante la permeabilidad creciente de la crisis de representación, concebida como el desgaste sistemático de los mapas cognitivos. Una base epistemológica de naturaleza postdisciplinar, permite contrarrestar la exclusión de métodos producto de la separación de las disciplinas. Todo ello, nos permite reconocer que, el objeto de la Educación Inclusiva ha cambiado -a pesar no estar claro, en cada una de sus periodizaciones-, no así sus métodos de aproximación y/o abordaje. Esto es, ¿cómo se hace? Es menester señalar que, la rigidez de las disciplinas deviene una práctica teórico-metodológica infértil, restringiendo la integración de aportes de diversa naturaleza en la construcción de nuevo saber o eje de 
tematización para sustentar la tarea de dicho enfoque.

Formativamente, todo ello acarrea problemas ligados a la enseñanza de la Educación Inclusiva. Un efecto que comúnmente he observado en la formación de los educadores a nivel de pre y post-graduación consiste en operar en referencia a una estructura de conocimiento inexiste y falsificada. Por inexistente, comprendo aquello que no ha sido construido -a pesar que en trabajos anteriores he explicado que el enfoque en discusión presenta un estatus preconstructivo, es menester reconocer que, sus articulaciones cognitivas se ubican en ideas, saberes y conceptos fuera de su estructura de conocimiento-. De este modo, observo que las coordenadas cognitivas que sustentan el actual discurso de la Educación Inclusiva producto del vacío epistémico que enfrentarecurren al legado de la Educación Especial -en su dimensión educativa- satisfaciendo dicha carencia. Todo ello, demuestra que su actual estructura de conocimiento, reproducida por las políticas públicas, $y$, especialmente, por la investigación educativa y la formación del profesorado, opera mediante mecanismos de falsificación epistemológica. En este contexto, si analizamos el encuadre cultural que delimita la abducción y utilización de determinados conceptos para crear condiciones de diálogo y comprensión en torno a su objeto, sentido $y$ alcance, opera en lo que he denominado como tropismo negativo, es decir, sus saberes y conceptos contribuyen a distorsionar su real objeto de conocimiento. Los conceptos que actualmente fundamentan la actividad de la Educación Inclusiva consolidan una gramática propiedad del individualismo metodológico. Si examinamos los conceptos en referencia a su estructura de conocimiento auténtica, observo que, éstos, son propiedad de la multiplicidad. Todo ello, es consecuencia de una metodología y de un corpus de decisiones inadecuadas. ¿Qué define las necesidades de estudio de la
Educación Inclusiva, y, especialmente, de los Estudios sobre Educación Inclusiva?

En lo que se refiere al campo de conocimiento de la Educación Inclusiva he explicado en anteriores trabajos, algunas de sus principales características, entre las cuales destacan: la elasticidad, la flexibilidad, la multiplicidad de entradas, su condición rizomática y la heterotopicalidad de temáticas y discusiones que en él son albergadas. Su funcionamiento opera en lo no-lineal, delimitando un campo de naturaleza polifonal. Este es un territorio atravesado por complejas condiciones de producción cuyo principio rector es la diversidad epistemológica articulada, metodológicamente, desde la hermenéutica diatópica y analógica. La primera, corresponde a la propuesta desarrollada por Sousa (2009), consiste en examinar en un plano de igualdad y equidad epistemológica dos o más saberes, conceptos, disciplinas, influencias, teorías o territorios, con el objeto de identificar las coincidencias, las mediaciones y las distinciones capaces de enriquecer el diálogo de saberes. En esta arista, coincide con lo expuesto por FornetBetancourt (1994), respecto de la hermenéutica pluritópica y la ética de la liberación, ámbitos temáticos significativos en el ensamblaje de un nuevo terreno para practicar los principios propuestos por la comprensión epistemológica de la Educación Inclusiva. La contribución de la hermenéutica pluritópica impacta en este trabajo, específicamente, en la ampliación de los sistemas de razonamientos, interesándose por articular una comprensión más amplia y críticamente subversiva acerca de la multiplicidad de perspectivas epistemológicas que confluyen en este campo de conocimiento, concebida en términos de red. Se interesa por crear sistemas de razonamientos capaces de penetrar en determinados patrones institucionales de funcionamiento de la sociedad, concebida como un dispositivo de cristalización de procesos de liberación. La ética de la liberación por su 
parte, articula su centro de actividad en la premisa que explicita el conjunto de condiciones de ininteligibilidad de lo diferente. Ambas se convierten en estrategias ético-políticas del campo epistemológico, trazando nuevas rutas de movilización del espacio escolar y sus procesos deontologizadores. La hermenéutica analógica creación del destacado filósofo iberoamericano Mauricio Beuchot, es concebida a efectos de este trabajo, como una estrategia analítico-metodológica que permite determinar los límites de verdad entre dos o más disciplinas, influencias, marcos teóricos y políticos y teorías que en él tienen lugar.

¿Cómo organizar una metodología que emerja desde una multiplicidad de disciplinas -como es el caso de la Educación Inclusiva-? Una primera reflexión consistiría en reconocer la necesidad de cambiar el camino de aproximación metodológica. Previo a ello, identificar que no existe un método consolidado en este campo de producción. Sin duda, esta interrogante, inscribe su ámbito de acción en lo post-disciplinar, recurre para ello, a la integración de aportes de diversa naturaleza, organizando su actividad en la producción de un nuevo recurso metodológico. Coincidiendo con Bal (2003) observo altamente fértil, la posibilidad de recurrir al potencial de los conceptos -concebidos como contraparte cultural- para explorar, develar y describir el repertorio metodológico en un campo emergente como es la Educación Inclusiva. ¿Cómo articular una alternativa diferente en materia metodológica? Sin duda, es necesario crear un terreno metodológico común

nuevamente emerge la noción en referencia a la base post-disciplinar-. Los conceptos son centrales en el trabajo epistemológico, una determinación y descripción analítica acertada promueve una comprensión más detallada y completa sobre su objeto. A pesar de reconocer que el objeto-misión de análisis-de la Educación Inclusiva constituye otro punto problemático, específicamente, al no existir claridad sobre el mismo. Frente a ello, cabe preguntarse acerca de qué movimientos críticos, influencias y marcos epistémicos y políticos van organizando su campo y objeto de conocimiento. Sin duda, esto, nos permite reconocer que, la Educación Inclusiva en tanto campo de conocimiento, es un espacio de confluencia de múltiples influencias e intereses. Por otra parte, identificar los mecanismos de encuadramiento de su método y objeto resultan acciones sustantivas en la comprensión epistemológica del mismo.

Finalmente, considero significativo reconocer el conjunto de elementos que definen el carácter programático del propio término de Educación Inclusiva. En tal caso, el conjunto de discusiones aquí matizadas, se convierten en antecedentes claves para identificar el tipo de argumentos y sistemas de razonamientos devenidos en definiciones, explicaciones y supuestos epistémicos y metodológicos ambiguos, abstractos y generales sobre su alcance y sentido. Sin duda la interrogante por el objeto de la Educación Inclusiva, con antelación analizará la diversidad de objetos de estudios que desarrollan las disciplinas que confluyen y participan del ensamblaje de su campo. ¿A qué pertenecen tales objetos de estudio?, ¿por qué no las constituyen juntas?, ¿qué estudia realmente la Educación Inclusiva?, ¿qué significa la 'educación' y la 'inclusión' en este marco?, ¿en qué se enfocan cada uno de ellos? Estas y otras interrogantes críticas intentaré responder a lo largo de este trabajo. Sin embargo, siempre el objeto participa en la producción de significados al interior de un enfoque particular.

Razón por la cual, prefiero concebir la Educación Inclusiva como un enfoque que no suscribe a ninguna práctica teórica o metodológica particular, sino que se construye a través del movimiento, el encuentro, la plasticidad y la constelación de saberes, métodos, objetos y teorías, formando un pensamiento temporal para problematizar los principales fenómenos 
educativos, trabajando en las intersecciones de las disciplinas.

\section{2.-CONSTELACIONES METODOLÓGICAS, MOVIMIENTOS DE SABERES Y PLASTICIDADES CONCEPTUALES IMPLICADAS EN LA COMPRENSIÓN EPISTEMOLÓGICA DE LA EDUCACIÓN INCLUSIVA}

La epistemología de la Educación Inclusiva desde su surgimiento ha sido tematizada y problematizada en referencia a un espacio relacional de múltiples objetos, problemas, métodos, objetivos y teorías que, mayoritariamente, estudian y se interesan por la comprensión del devenir humano y sus problemáticas -en sí mismas, patologías sociales crónicas de la humanidad-, enmarcadas sobre un eje crítico-transformativo del pensamiento, documentan que, dicha temática ha sido invisibilizada por los investigadores y sus agendas, un tema no discutido con la debida pertinencia en las propuestas, políticas y programas de formación del profesorado a nivel de pre y post-graduación en el mundo, producto de un amplio espectro de equívocos de interpretación y aproximación a su objeto, mediados por una extraña política intelectual. EI conocimiento de la Educación Inclusiva expresa una naturaleza diaspórica, reticular, micropolítica -analítica y epistémica-, post-disciplinar; es sinónimo de transformación -construye su cuerpo de saberes y actúa por fuera del modelo dominante-, deconstrucción y creación de otros mundos. Es una expresión post-crítica y postdisciplinar.

Emerge desde un pensamiento temporal, que va definiendo sus lugares -topografía y topología epistémica- de configuración -fases de periodización y gramáticas- y producción de sentido -dimensión hermenéutica-, a través de patrones de movimiento y desplazamiento -surgidos en un espacio de tipo diaspórico y rizomático, es decir, un campo de conocimiento con múltiples entradas -compuesto por principios metodológicos, tales como: a) el funcionamiento diaspórico, b) el viaje como recurso comprehensivo, c) la heterogeneidad y la multiplicidad de sus cuerpos, disciplinas e influencias, d) la elasticidad y el conexionismo, e) la constelación, el movimiento y el encuentro, f) el énfasis en la exterioridad, g) la interseccionalidad epistémica, h) la multiplicidad, i) la ruptura/ emancipación, j) la cartografía y la orientación, etc.-. Sus desplazamientos se ensamblan bajo la lógica del dispositivo, confluyendo en un espacio de encuentro denominado por Pratt (1992) como zona de contacto y, posteriormente, sometiendo dichos cuerpos de saberes e influencias organizativas a traducción científica y a examen topológico. Es decir, vertidos en un batido centrífugo desde el cual se depuran nuevas unidades intelectuales y conceptuales. ¿Cuáles son los objetos, los métodos y las teorías entre las cuales se moviliza y opera la comprensión epistémica de la Educación Inclusiva?, ¿bajo qué formas metodológicas, se expresa la Educación Inclusiva en tanto eje de movilización de las fronteras configurantes de la Ciencia Educativa?, o bien, ¿mediante qué elementos, se dibuja el pensamiento fronterizo de la Educación Inclusiva?

De acuerdo con esto, es posible afirmar que, el pensamiento epistemológico de la Educación Inclusiva es el 'pensamiento de la relación' (dispositivo-ensamblaje), del 'movimiento epistémico de saberes, conceptos y métodos' (diáspora epistémica) y del 'encuentro' (zona de contacto y producción de lo nuevo). El ensamblaje y su arquitectura epistémica, operan mediante la movilización de las fronteras de las disciplinas, confluyendo en una zona de contacto, cuya interrelación fabrica una 'frontería epistémica', es decir, una zona de indeterminación, de apertura, de creación, de un tercer espacio, denominado 'heterotópico', coherente con su naturaleza post-disciplinar. 
Es un espacio social, educativo y político de la monadología. La construcción del conocimiento de la Educación Inclusiva, se orienta a la superación de las estrategias de aplicacionismo epistémico devenidas en la institucionalización de mecanismos de fijeza, anquilosando los saberes, los métodos y las disciplinas al dictamen del pasado y con ello, al círculo de reproducción. El conocimiento auténtico de la Educación Inclusiva -de naturaleza post-disciplinar- apela a la ruptura de los límites de las disciplinas, trabaja en la producción de lo nuevo, razón por la cual, la tematización y localización de sus saberes constituye una tarea compleja y de amplio alcance. La complejidad de su objeto queda definida por su naturaleza post-disciplinar, encontrando su organicidad y eficacia en un saber que no le pertenece a nadie, enfatizando en la producción de lo nuevo. Un desafío metodológico consiste en determinar los medios de configuración de su objeto auténtico y los medios de orientación en el tipo de pensamiento intelectual que ésta forja.

La comprensión y construcción epistemológica de la Educación Inclusiva, exige movilizar las fronteras de la Ciencia Educativa. En efecto, todas estas discusiones puntualizan en el estudio de los ejes de estructuración del 'espacio diaspórico de la inclusión' -en tanto arquitectura epistémica-. Recurriendo a la metáfora propuesta por Ocampo (2016) "el conocimiento de la Educación Inclusiva se construye diaspóricamente", es decir, a través de una dispersión creciente de saberes que posibilitan su ensamblaje epistémico, operando mediante un conjunto de 'movimientos', 'desplazamientos' y 'encuentros' particulares entre diversas disciplinas, influencias teóricas, campos de conocimiento y geografías epistémicas. La diáspora epistémica (Ocampo, 2016) -referida a las operatorias del saberposibilita la determinación de una multiplicidad de tipos de movimientos -claves en la configuración de su campo de conocimiento-, mediante los cuales se diversifican los saberes y se insertan en un nuevo mecanismo de ensamblaje. Para ello, es menester comprender la multiplicidad de temporalidades que confluyen en dicha espacialidad, insertándose en determinados períodos y forjando una gramática particular -rejilla de ideas-. En suma, el saber de la Educación Inclusiva es propio de la historicidad del presente.

El conocimiento de la Educación Inclusiva, claro está, no pertenece, ni encuentra su legado en la imposición epistémica y pragmática de la Educación Especial -donde está última, carece de una comprensión epistémica, a pesar de evidenciarse números esfuerzos reflexivos al respecto, no hay claridad sobre su origen y naturaleza en relación a su conocimientoconstituye uno de los fracasos cognitivos (Spivak, 2008) más evidentes; además, de promover un efecto de hibridación y falsificación de sus cuerpos de saberes, lenguajes y condiciones pedagógicas e interpretativas. Por el contrario, si los investigadores han redundado en este error, ha sido única y exclusivamente, por su incapacidad para aproximarse a su real objeto, devenido en la consolidación de una extraña política de producción intelectual que fundamenta este campo, así como, de una falsa política de transformación del mundo y deconstrucción del conocimiento, incapaz de responder a la pregunta: ¿en qué sentido la Educación Inclusiva es para todo el mundo? La comprensión epistemológica de la Educación Inclusiva se concibe en términos de un sistema de actualización y transformación permanente del conocimiento estructurante y estructurador de la Ciencia Educativa. Por esta razón, ha sido denominada como un dispositivo macro-educativo. Su interés se posiciona en el estudio de las condiciones de producción y/o fabricación del conocimiento, es un saber que deambula, viaja, recorre y transita por una amplia multiplicidad de campos del conocimiento, surge del encuentro, de la constelación, del movimiento, de la conexión y de la producción de lo nuevo. Su naturaleza indisciplinar, manifiesta la necesidad de avanzar en la construcción de sus saberes auténticos, es decir, superar el efecto falsacionista e híbrido que afecta a sus 
cuerpos de conocimientos empleados en la actualidad.

¿Cuáles son las innovaciones que plantea la epistemología de la Educación Inclusiva? Una primera aproximación consiste en afirmar el tipo diferente de objeto que construye, además de develar la naturaleza de éste, desde una perspectiva post-disciplinaria, concebido como un campo adverso a los enfoques educativos tradicionales. Posee la capacidad de articular un repertorio metodológico que emerge desde lo mejor de cada una de las disciplinas que confluyen en su organización. Por otro lado, contribuye a reconocer que la 'inclusión' y la 'Educación Inclusiva', expresan un carácter programático. La centralidad de las innovaciones que plantea esta comprensión epistemológica, consiste en acceder a la realidad educativa mediante conceptos que permitan operativizar la construcción de una nueva espacialidad y formatos de justicia educativa. Asume la cuestión metodológica como una tarea central en este enfoque, desafía las disciplinas y los marcos disciplinarios heredados, intentando producir algo nuevo, a pesar de no encontrarse fijo en ninguna disciplina, se refugia en la combinación de una multiplicidad de métodos, objetos, teorías, objetivos, influencias, sujetos y territorios a fin de aflorar algo nuevo. Si bien es cierto, la producción de lo nuevo es central en esta comprensión epistemológica, no obstante, su fuerza analítica se orienta a ofrecer una mejor comprensión del fenómeno a nivel político, teórico, metodológico, ético y pedagógico, con la finalidad de comprender mejor su objeto.

Quisiera insistir en el reconocimiento sobre la complejidad que afecta a la definición del campo de la inclusión -en sus diversas dimensiones-, lo mismo ocurre con la comprensión de su objeto y método. Este es un campo que propicia el encuentro entre varios métodos, disciplinas, saberes, teorías y objetos.
La construcción epistemológica de la Educación Inclusiva expresa un patrón de funcionamiento basado en el encuentro y en el movimiento, cuyo ensamblaje -a través de la lógica del dispositivo (Foucault, 1973; Deleuze, 1990; Agamben, 2006)-, constituye en palabras de Bal (2016) un pensamiento temporal que emerge en el movimiento. Por tanto, afirmar que, la Educación Inclusiva es una teoría sin disciplina, no sólo refiere a su naturaleza postdisciplinar y a la producción de lo nuevo ${ }^{2}$, sino más bien, al conjunto de mecanismos de reorganización de la Ciencia Educativa y de la pedagogía, desde las afueras de las disciplinas. Esto es, un saber que no le pertenece a ninguna disciplina, que debe avanzar en la configuración de la autenticidad de su objeto y de sus cuerpos de conocimientos, así como, ofrecer respuestas al problema del método. En suma, la epistemología de la Educación Inclusiva es la epistemología de la Ciencia Educativa en el siglo XXI, puesto que, moviliza un conjunto heterogéneo de transformaciones en cada una de sus disciplinas configurantes -las refunda-. A este sistema de actualización permanente -pensamiento temporal- lo he denominado 'dispositivo macro-educativo' sistema de recognición-. Metodológicamente, es un conocimiento que emerge de la extracción de los aportes más significativos -diaspóricos y viajeros- de sus campos de conocimientos y objetos confluyentes, cuya operación constituye la clave para avanzar en la construcción de sus saberes auténticos. Su naturaleza 'in-disciplinar' -no fija en ninguna disciplina- exige también, la creación de nuevos modos de lectura y producción en el abordaje de sus fenómenos.

En suma, afirmaré que está construcción epistemológica emerge desde una amplia y extensa constelación de ideas, saberes, preocupaciones, métodos, sujetos, territorios,

$2 \quad$ Refiero a la capacidad de producir sus saberes auténticos, mediante los cuales es posible transformar los modos de análisis y praxis de la educación a través de una nueva espacialidad. 
teorías e influencias intelectuales y metodológicas -no visibilizadas y comprendidas hoy por sus investigadores-, conformando un espacio diaspórico de producción y análisis, ratificando la necesidad de avanzar en la construcción de una metodología de saberes auténticos del enfoque -ejes gravitantes en la creación del nuevo marco de sustentación teórica exigida por el campo de conocimiento auténtico de ésta - Confluyen en esta espacialidad analítica una multiplicidad de saberes, métodos, objetos, teorías y temporalidades. De ahí, que ésta sea una epistemología de la heterocronía -de la confluencia de múltiples tiempos en el estudio de un mismo fenómeno-. La construcción epistemológica de la Educación Inclusiva no puede ser examinada sin referir a la 'experiencia migratoria' y los 'ejes de movilidad y desterritorialización' que afectan a sus saberes y principales geografías epistémicas que participan en el ensamblaje de su campo de conocimiento auténtico -de naturaleza postdisciplinar-.

Es menester señalar que, a pesar de existir una serie de problemáticas por parte de los investigadores para aproximarse al real objeto de la Educación Inclusiva y de la omisión permanente de su historia intelectual, es posible observar tres grandes campos de conocimiento en su incipiente desarrollo teórico -determinados según períodos particulares-. Tales campos son: a) falsificado ${ }^{3}$ (configurado predominantemente a la partir de la insistencia por el esencialismo y del individualismo metodológico, la imposición del modelo epistémico y didáctico tradicional de la Educación Especial, metáfora de mayor operatividad procedimental -invisible y omitida en los debates intelectuales- inclusión a lo mismo y los esfuerzos acomodacionistas en términos teóricos, políticos y prácticos), b) mixto, híbrido o travestizado 4 (articulado por fuerzas que determinan la elasticidad del campo, la convergencia de una amplia multiplicidad de influencias, aportes teóricos y opciones metodológicas que operan mediante un funcionamiento diaspórico, es decir, saberes y fragmentos de información repartidos por distintos campos intelectuales y disciplinas, el cruce epistemológico cuya examinación se posiciona desde el aplicacionismo epistémico y en desconocimiento de la demanda teóricoepistemológica auténtica de este campo, y, la ambigua demanda teórica asociada al enfoque -eminentemente travestizada-) y auténtico (estructurado a partir de los supuestos y axiomas epistemológicos centrales otorgados por la episteme de la inclusión, surge desde el reconocimiento de su naturaleza postdisciplinar, la creación de una metodología de examinación crítica de los desplazamientos y viajes de sus instrumentos conceptuales y el interés metodológico por la creación de saberes propios del enfoque).

Entre las principales condiciones de producción epistemológicas de la Educación Inclusiva, destacan: a) la diseminación, b) el carácter diaspórico y c) la dimensión extra-teórica. Su orden de producción, es decir, el conjunto de leyes internas que definen su funcionamiento, alcanza su máxima expresión en la diseminación, es decir, en la dispersión, explicitando un conjunto de enredos genealógicos de dispersión como parte de las condiciones de configuración de su campo. Su formación depende de la confrontación de diversas decisiones epistemológicas y metodológicas -es un campo del disenso epistemológico-, reclama la construcción de una metodología sensible a los procesos de fabricación de su conocimiento especializado.
$3 \quad$ Corresponde al campo hegemónico, dominante y celebratorio, cuya particularidad encierra su funcionamiento y objetivos analíticos y de lucha en el centro de capitalismo. De ahí que, el autor de este documento en otros de sus trabajos afirmará que existe una visión de inclusión alojada al interior del capitalismo, el imperialismo y el colonialismo.
$4 \quad$ Corresponde al momento intelectual actual de la Educación Inclusiva. 


\section{3.-SOBRE LAS CONDICIONES DE PRODUCCIÓN DE LA EDUCACIÓN INCLUSIVA}

El abordaje de las condiciones de producción constituye una propiedad inherente a toda actividad científica, es decir, desempeña una parte sustantiva del centro crítico de un determinado campo de conocimiento. El estudio de las condiciones de producción no es otra cosa que un mecanismo de descripción analíticometodológica sobre las condiciones que crean y garantizan la producción y el funcionamiento del conocimiento según la naturaleza epistemológica de un determinado fenómeno. Al transferir esta conceptualización al estudio epistemológico de la Educación Inclusiva, observo que, sus condiciones de producción quedan definidas transversalmente por un orden de producción de tipo diseminal, el diaspórismo epistemológico y la dimensión extra-teórica como pieza angular en la producción de su fenómeno. Todas estas condiciones son albergadas en una base epistemológica de naturaleza post-disciplinar.

En anteriores trabajos he afirmado que el conocimiento de la Educación Inclusiva se construye diaspóricamente, es decir, mediante una dispersión de saberes que trazan una movilidad no-lineal incesante que atraviesan multiaxialmente diversos campos, discursos, métodos, objetos e influencias. La diáspora epistémica concebida como una de las modalidades de construcción del conocimiento de la Educación Inclusiva, facilita el reconocimiento de un centro crítico ensamblado a partir de un conjunto de enredos genealógicos de diversa naturaleza. La conformación de un espacio diaspórico opera como una constelación de ideas, intereses, posiciones, geografías epistémicas, objetos y teorías, modelan diversos movimientos, puntos de encuentro y sistemas de relación a, los cuales dialogan, conexionan, visitan, movilizan, interseccionan, colisionan y constelan entre determinados puntos de captura y/o forclusión.
Tanto las condiciones de producción como las condiciones de posibilidad vinculadas al estudio de la Educación Inclusiva acontecen en el marco de un encuadre cultural particular. En él, emergen y son legitimadas determinadas categorías que van modelizando el funcionamiento y la comprensión de la realidad. Las primeras, aluden al conjunto de mecanismos implicados en la fabricación del conocimiento especializado de un determinado campo de estudio, mientras que, las segundas, por su parte, en palabras de Canguilhem (1975) constituyen un encuentro de series, delimitando el campo práctico en el que va configurándose un determinado problema, incluye dimensiones políticas, sociales, económicas, culturales, etc. En otras palabras, el encuadre cultural es aquello que sostiene a la noción de condiciones de posibilidad, constituyendo un eje crucial en el estudio de las periodizaciones y gramáticas intelectuales y sus objetos de conocimiento en determinadas instancias del pensamiento educativo o desarrollo intelectual del enfoque.

Las condiciones de posibilidad pueden aplicarse al estudio del objeto de conocimiento de la Educación Inclusiva, o bien, devenir en una estrategia de integración del conocimiento de dicho enfoque en la estructura social y, especialmente, en el sistema-mundo. Desde una perspectiva kantiana, es posible emplear las 'condiciones de posibilidad' en tanto dispositivos que permiten la emergencia y/o funcionamiento de su objeto, "de tal manera que ésta ya no tenga sólo relación con el análisis de las condiciones de posibilidad de conocimiento del objeto, sino que igualmente abarque el análisis de las condiciones de posibilidad del objeto de conocimiento" (Vandenberghe, 2004, p.317). Para ello, es fundamental promover un análisis que ahonde en el conjunto de dimensiones socio-históricas implicadas en la emergencia del concepto y de su comprensión epistemológica en tanto conocimiento educativo que plantea innovaciones complejas a nivel estructural. El 
estudio de las condiciones de posibilidad no se encuentra disociado del examen epistemológico de la Educación Inclusiva. Las condiciones de posibilidad delimitan a juicio de Bowman (2008) capacidad para articular formas de intervención -previamente delimitadas por las fuerzas de un determinado encuadre cultural-, leyéndose como una "red de diferencias disciplinarias, $y$ frente a (el carácter constitutivo de) la disciplina y la diferencia disciplinaria, lo que ha surgido es enclaves disciplinarios, ininteligibilidad mutua y desarticulación" (Bowman, 2008, p.93).

La post-disciplinariedad concebida como una condición de producción central del campo epistemológico de la Educación Inclusiva plantea condiciones de (des)rearticulación entre los diversos legados disciplinarios, influencias, teorías, objetos y métodos que participan en la configuración de dicho campo. Se propone alterar los discursos disciplinarios y las condiciones de fijeza al pasado, así como, sus mecanismos de producción del conocimiento. Su estrategia consiste en el despliegue de condiciones que permitan intervenir en las configuraciones específicas de los espacios disciplinarios de producción y legitimación del saber. ¿Qué compromisos disciplinarios podrán tener lugar al interior de este campo de producción?, ¿bajo qué condiciones, tales compromisos son deslindados, transformados o subvertidos?

De acuerdo con Derrida (2003) es imposible que un espacio disciplinario -cerradura epistémica- exista en tanto campo de batalla, debido a la ausencia de un locus de verdad fuera del mismo (Bowman, 2008), determinando la presencia de una multiplicidad, heterogeneidad y heterotopicalidad de elementos. Por consiguiente, el estudio de las condiciones de producción inquiere en un análisis acerca de la multiplicidad de demandas de legitimidad que tienen lugar al interior del campo de la Educación Inclusiva. Retomando los aportes de Mowitt (1992), observo fértil establecer la diferenciación entre 'objeto mismo de la argumentación' y 'objeto disciplinario'. El primero, a juicio de Bowman (2008) deviene en una construcción institucional específica, mientras que el segundo, alude al conjunto de decisiones a través del cual se contraponen los enfoques teóricos contra sus compromisos políticos. En tal caso, la postdisciplina permite la reconfiguración -e incluso, promueve condiciones de defabricación- del saber, sus mecanismos de institucionalización y formaciones paradigmáticas. Las condiciones de posibilidad han de ayudarnos a visibilizar el conjunto de formas constructivas en torno al objeto de su argumentación y su campo propio de argumentación (Arditi, 2007).

De acuerdo con esto, "una disciplina es algo que se constituye fundamentalmente como una estructura hegemónica rivalizada congregada alrededor o en el nombre de un objeto particular" (Bowman, 2008, p.97). ¿Qué pasa entonces con lo disciplinario en el contexto de producción de la Educación Inclusiva? Tal como he explicado en "Las políticas de la mirada y la comprensión epistemológica de la Educación Inclusiva: ¿en qué sentido la Educación Inclusiva es para todo el mundo?", este campo deviene en el ensamblaje de una red reticular compuesta por pliegues de diversa naturaleza, albergando una amplia multiplicidad de disciplinas, discursos, objetos, influencias, marcos teóricos y políticos. Su dimensión post-disciplinar observa en ellos, aportes significativos que permiten avanzar en la construcción de un nuevo saber, sometiéndolos a traducción. Por tanto, este campo se moviliza por cada uno de estos aportes, no se queda fijo en ninguno de ellos, permitiéndonos afirmar que, la Educación Inclusiva, no suscribe a ninguna práctica teórica y metodológica en particular.

En relación a la dimensión diseminal, es preciso destacar que, ésta, es propiedad constitutiva y central del orden de producción de la Educación Inclusiva. El orden de producción en términos foucaultianos alude al conjunto leyes o propiedades de funcionamiento de 
un determinado campo de conocimiento. En reiteradas oportunidades he comentado que este campo opera mediante un conjunto de tecnologías de dispersión y diseminación, propiedades en constante movimiento de carácter no-lineal, caracterizado por confluir en él, pliegues e itinerarios no-lineales de diversa naturaleza. La dimensión extra-teórica junto al orden de producción, constituyen dos de las condiciones de producción más significativas vinculadas al estudio epistemológico de la Educación Inclusiva. La dimensión extra-teórica permite reconocer que este es un fenómeno modelizado por fuerzas sociales, políticas, culturales y estructurales, las que van matizando su sentido y tópicos de análisis. Por tanto, son las características de los propios fenómenos sociales las que regulan el funcionamiento de su campo de producción mediante leyes propias del fractal.

\section{4.-LAS ESTRATEGIAS DE CONSTRUCCIÓN DEL CONOCIMIENTO DE LA EDUCACIÓN INCLUSIVA}

La noción 'estrategias de construcción del conocimiento de la Educación Inclusiva', aparece por primera vez en el capítulo: "Gramática de la Educación Inclusiva: ejes críticos para cartografiar sus condiciones de producción y funcionamiento epistemológico", en él, explico que, éstas constituyen una pluralidad de mediaciones estratégicas, a través de las cuales, es posible comprender cómo se va configurando el conocimiento especializado de la Educación Inclusiva. Debido a sus particularidades, éstas, se albergan en el núcleo analítico de la diseminación y del movimiento, propiedades inherentes a la comprensión epistemológica aquí discutida.

Las estrategias de construcción del conocimiento de la Educación Inclusiva operan en un espacio diaspórico, concebido como una constelación de ideas, intereses, posiciones, geografías epistémicas, objetos y teorías que confluyen en una espacialidad rizomática y en un ensamblaje basado en la lógica del dispositivo; modelan diversos movimientos y puntos de encuentro, constituyendo de esta forma, la producción de su campo intelectual. Es un 'espacio de encuentro' entre una amplia, extensa y rica multiplicidad de saberes, campos de conocimientos, objetos, métodos, objetivos y teorías que conectan, dialogan, visitan, movilizan, interseccionan, colisionan y constelan. Es un campo caracterizado por la heterotopicalidad. En este espacio tienen lugar diversos mecanismos de funcionamiento epistemológicos, analíticos y metodológicos, procesos de fabricación, culturas epistémicas que crean y garantizan el conocimiento, regiones y geografías del conocimiento que producto de sus intersecciones epistémicas generan nuevos conceptos, procesos de formación y movilidad de conceptos y estrategias de construcción del saber epistémico.

Entre las principales estrategias de construcción del conocimiento que circulan en el campo de producción de la Educación Inclusiva destacan: a) préstamos epistémicos, b) injertos, c) sustitución, d) deslocalización, e) reciclaje, f) ensamblaje condición transversal- g) y trasmigración. En este apartado, me referiré a algunas de ellas, sin abusar de la extensión editorial establecida por la revista. Es menester recordar que, es el carácter diseminal el que atraviesa cada una de las estrategias aquí mencionadas.

El injerto es una de las principales estrategias de construcción del conocimiento de la Educación Inclusiva -estructura falsificada e híbrida-. Según Derrida (1997), este no sobrevive a lo propio de la cosa, es factor de mutabilidad, es propiedad del collage, remite a mecanismos de reinscripción de saberes, tal como sugiere el filósofo francés, "se forman de una a otro, se contaminan en su contenido, tienden a veces a rechazarse, pasan elípticamente de uno a otro 
y se regeneran allí en la repetición, en el hilado de un sobrehilado" (Derrida, 1997, p.533-534). La injerción de saberes, discursos y métodos, por ejemplo, posee la capacidad transformarlos, fabricando una nueva imagen o funcionalidad. Sobre este particular, Derrida (1997) insiste señalando que, "cada elemento injertado continua irradiando hacia el lugar de su toma, lo transforma así, al afectar al nuevo terreno" (p.534). Es menester, develar los efectos de operación que tienen lugar en este campo de producción, genera cambios en las formas de funcionamiento del saber. Para el epistemólogo francés, es el único método que posibilita resolución de este tipo de contradicciones. Cada una de las estrategias de construcción del conocimiento, definen la forma en la que este podrá moverse, ¿cuáles son las formas de movimiento que permiten comprender, caracterizar y acceder a la resolución de diversos tipos de contradicciones que tienen lugar en dicho campo?

Cada una de estas estrategias comparte la condición de trasplante, acción que es múltiple, recoge aportes procedentes de diversos campos, injertando lugares, vocabularios, ideas y saberes, mediándose en cada ocasión por las condiciones de exportación, deviene en un campo que funciona mediante diversas operatorias, actúa en la des- y en la rearticulación, encuentra su funcionamiento en la integración -no en el aislamiento-. Mediante su actividad es posible reconocer la singularidad de cada uno de sus elementos. El campo epistemológico de la Educación Inclusiva se convierte en un campo de lo 'infinito', en permanente movimiento y captura/ apertura hacia rumbos desconocidos. En él, son albergados recursos de diversa naturaleza, describe así, la existencia de un movimiento incesante de sustitución colocando en evidencia la colaboración de un marco que monta y desmonta, es decir, define lo que queda dentro o fuera. No intenta mostrarse en la constitución de ciertas consecuencias epistemológicas a las que conducen sus sustituciones, formas $y$ transformaciones. Los elementos participantes de este campo se encuentran desconectados, deshilados, esto constituye una de sus principales formas de fracaso. Este es un campo que va modelizándose a través de ciertos enredos genealógicos de dispersión, cruce de caminos epistemológicos de diversa naturaleza y magnitud. Es un campo epistemológico de singularidades múltiples.

Una de las estrategias más comunes se reduce al aplicacionismo epistémico, este consiste en el atrapamiento del fenómeno en los marcos, valores y concepciones de un determinado modelo de interpretación. A través de él, proliferan nuevas modalidades de hibridación -esta, no es de exclusividad estratégica-. El aplicacionismo epistemológico opera negando la autenticidad del fenómeno, atrapa y captura el objeto en su externalidad. Su principal dificultad deviene en prácticas de transposición analíticometodológicas mutiladas, restringiendo y ocultando la autenticidad del fenómeno. Este es uno de los principales problemas junto a la hibridación que enfrenta la comprensión epistemológica, en específico, las condiciones de producción de la Educación Inclusiva. Apela por la cristalización de un saber y de condiciones de producción estratégicas, que legitima la normatividad y la interioridad epistemológica -reproducción-, inaugurando un sistema de construcción artificial de saberes. El aplicacionismo epistemológico es, uno, de los múltiples factores que convergen en el estudio de los mecanismos de falsificación de saberes que enfrenta la Educación Inclusiva.

Otras estrategias de construcción del conocimiento de la Educación Inclusiva son el préstamo y la sustitución, estrategias de interioridad epistemológicas; en sí mismas, expresiones del aplicacionismo epistemológico. Los injertos aluden a mecanismos de aleación, unión entre dos o más elementos, del que 
germinan nuevas modalidades de saberes, a través de sistemas de saberes, teorías, conceptos y disciplinas -preferentemente- de naturaleza isomórfica. Sus mecanismos de operación no quedan cerrados a dicha normatividad, sino que, pueden alcanzar mecanismos de producción entre campos y saberes de carácter extra-disciplinar -principal propiedad del campo epistemológico de la Educación Inclusiva-. Los reciclajes epistémicos, por su parte, devienen en un proceso de re-utilización de saberes y recursos metodológicos preferentemente- con el objeto de ampliar y extender sus usos y efectos, previa traducción y examinación topológica.

Por consiguiente el campo de conocimiento de la Educación Inclusiva se convierten en

[...] el mapa es abierto, es conectable en todas sus dimensiones, desmontable, reversible, susceptible de recibir constantemente modificaciones. Puede ser roto, invertido, adaptarse a montañas de cualquier naturaleza, ser comenzada su realización por un individuo, grupo, formación social. Se le puede dibujar en un muro, concebirlo como una obra de arte, construirlo como una acción política o como una meditación. Puede ser uno de los caracteres más importantes del rizoma, tener siempre múltiples entradas (Deleuze y Guattari, 1970, p.34).

El estudio del campo de conocimiento de la Educación Inclusiva no sólo puede ser abordado en términos de sus principales características antes mencionadas, tales como, la elasticidad, la multiplicidad de entradas a la comprensión de su fenómeno, el funcionamiento diseminal y sus raíces rizomáticas, etc. Sin embargo, éstas, se convierten en propiedades centrales del mismo. No obstante, el estudio del campo de conocimiento puede serabordado analíticamente en referencia a las leyes de pensamiento que esta consolida progresivamente a través de su desarrollo. En tal caso, la identificación de diversas gramáticas de pensamiento y sus sistemas de razonamientos legitimados en el marco de una determinada periodización, se convierten en poderosas estrategias de análisis para develar las leyes de pensamiento, el tipo de objeto y sus modalidades de acceso a éstos. En este marco, he identificado tres tipos campos intelectuales consolidados y relevantes en el estudio teórico y metodológico de la Educación Inclusiva. El primero, de naturaleza falsificada, cuyos ejes de funcionamiento recurren a saberes, conceptos y herramientas metodológicas que no se encuentran en sintonía con la naturaleza de su base epistemológica -post-disciplinar-. Este es el campo hegemónico en el que la Educación Inclusiva articula su actividad científica, específicamente, producto de la ausencia de una estructura de conocimiento clara, coherente en criterios y principios científicos, recurre en su variante pedagógica a la imposición del modelo epistémico y didáctico tradicional de Educación Especial. El segundo, de carácter hibridizado o mixto, corresponde al entrecruzamiento de saberes, herramientas metodológicas y conceptuales, abduciendo en forma desmedida aportes de diversas disciplinas y campos del conocimiento. Al carecer de condiciones de legibilidad científica, este genera mecanismos de contaminación y travestización epistemológica. Finalmente, emerge el campo auténtico, de base post-disciplinar, no fijo en ninguna disciplina, más bien, cruza y atraviesa una amplia multiplicidad de elementos que confluyen en su ensamblaje. Este campo puede ser concebido en términos de una malla de conexión reticular, compuesta por elementos de diversa naturaleza. Su interés se orienta a la producción de un marco político, epistémico y metodológico de la multiplicidad.

El esquema que se presenta a continuación, se propone identificar la base epistemológica de cada uno de los tres campos identificados en el desarrollo intelectual de la Educación Inclusiva, 
los cuales, pueden sintetizarse de la siguiente

manera:

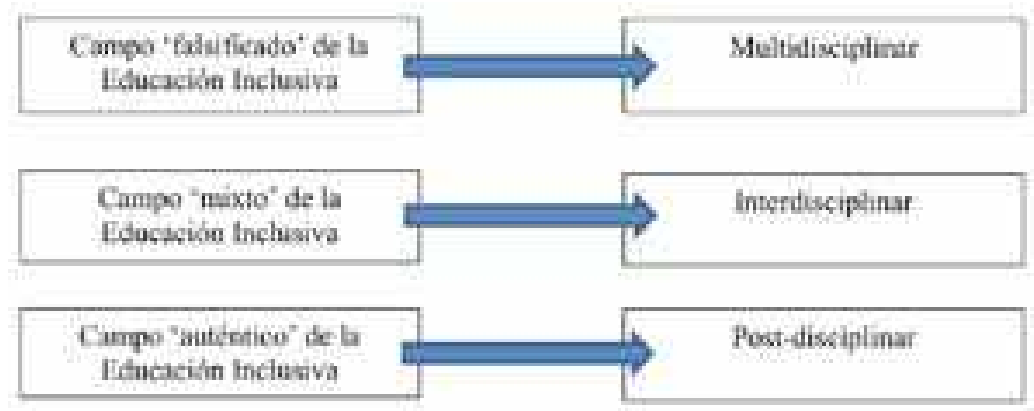

Figura 1. Síntesis de campos identificados en el desarrollo intelectual de la Educación Inclusiva.

Fuente: Elaboración propia.

\section{5.-EL UNIVERSO CONCEPTUAL DE LA EDUCACIÓNINCLUSIVA:UNAGRAMÁTICA DE LA MULTIPLICIDAD}

Los conceptos según Bal (2002) poseen la capacidad de articular condiciones de entendimiento, diálogo y comunicación entre elementos de diversa naturaleza, constituyen parte significativa del trabajo epistemológico, convirtiéndose así, en herramientas de intersubjetividad. Mediante su actividad ingresamos en la realidad, nos ubicamos en ella tomando posición. Los conceptos poseen la capacidad de deformar, desestabilizar o distorsionar un determinado objeto de conocimiento. Cuando la estructura teórica que los alberga es clara y se encuentra bien definida, estos, generan condiciones de inteligibilidad. En tanto, herramientas epistemológicas, poseen según Bal (2002) la capacidad de fundar un campo de conocimiento, a pesar que éstos, no se encuentran fijos en ninguna disciplina particular. Su naturaleza es propiedad del movimiento. En su paso por diversas disciplinas y campos de estudio, van mutando, dislocando y alterando sus significados. Los conceptos tienen una forma de sobrevivir a diversos fenómenos, cambian continuamente, tal como se modifica el saber que constituye epistemológicamente la inclusión. ¿Es, éste, un campo que procede por conceptos?, ¿para qué sirve la creación de conceptos y el trabajo con conceptos?, ¿qué formas metodológicas y analíticas participan en la producción de sus conceptos?

Epistemológicamente, los conceptos poseen la capacidad de determinar la práctica teórica de un campo de conocimiento, articulando diversas modalidades de focalización. En este trabajo abordo el estudio de los conceptos concebidos como recursos epistémicos y a su vez, metodológicos. En efecto, comparto con Bal (2002) la premisa de reemplazar los métodos por los conceptos, especialmente, en campos emergentes, en los que el investigador no tiene en que apoyarse -campos emergentes o de base post-disciplinaria-. El estudio de los elementos que configuran el universo conceptual de la Educación Inclusiva se plantea como objeto explorar las condiciones de producción, la sustentación de su gramática, así como, sus estrategias de construcción y ejes que pueden producir algún tipo de distancia, inviabilidad, inconsistencia o bien, ininteligibilidad respecto a su base epistemológica. Como puntapié inicial, afirmaré que, la gramática epistemológica de la Educación Inclusiva se sustenta en la multiplicidad, es decir, los conceptos de heterogeneidad, diferencia, diversidad, otredad, singularidad, pluralismo y así, un largo etcétera; son expresiones que progresivamente quedan albergadas en dicha gramática.

Desde la filosofía deleuzina y guattariana, los 'conceptos' constituyen una de las principales 
tareas de la filosofía, por esencia, ésta, es la disciplina orientada a la creación de conceptos, por ende, la creación de nuevos objetos. Los conceptos son propiedad de la creación, no de un estado finalístico. Sobre este particular, insisten señalando que, "tienen que empezar por fabricarlos, crearlos, plantearlos y convencer a los hombres de que recurran a ellos" (Deleuze y Guattari, 1990, p.4). Una de las demandas teóricas de la Educación Inclusiva expresa la necesidad de crear los conceptos, ¿por qué razón la inclusión no ha creado sus propios conceptos, a pesar que cada disciplina tiene la capacidad para engendrar los suyos propios? Los conceptos son una creación singular, se conoce la realidad mediante conceptos determinados. Frente a ello, cabe formular las siguientes interrogantes: ¿cuáles son los conceptos de ingreso en su campo epistemológico?, ¿cuáles son los conceptos de ficcionalización, transformación, nocivos, de autenticidad epistemológica, de focalización de su objeto, etc.?, ¿cómo se confrontan cada uno de ellos? y ¿cuáles son las estrategias de construcción de cada concepto?

Los conceptos no vienen dados, han de ser creados por cada campo de conocimiento, siguiendo sus condiciones de producción, que en el caso del campo aquí discutido, operan en la diseminación, el diaspórismo, lo extrateórico, etc. Los conceptos se convierten en recursos significativos en el estudio de los ejes de formación del campo de conocimiento. Deleuze y Guattari (1990) indican que al crear los conceptos, éstos, se plantean asimismo en términos de autoposición o un carácter autopoiético. Cuanto más creado es el concepto, más se plantea a sí mismo. Estas ideas nos llevan a asumir el estudio de los conceptos en tanto realidades filosóficas.

Los conceptos concebidos como herramientas intersubjetivas que facilitan el diálogo y la comunicación entre diversos campos del conocimiento, delimitan un marco interpretativo, se convierten en estrategias de intermediación entre diversos campos del conocimiento que confluyen, crean y garantizan el saber especializado de la Educación Inclusiva, conectan campos alejados en su actividad científica a la educación, reconociendo en ellos, aportes significativos y relevantes en la configuración de sus territorios -dimensión extra-disciplinar-. Los conceptos concebidos como estrategias de intermediación son asimilables a la lógica del dispositivo, es decir, logran capturar una multiplicidad de elementos significativos en la producción/funcionamiento de dicho campo. Los conceptos en sí, constituyen un tipo de contexto epistemológico, orientan la mirada y el entendimiento hacia una forma particular de concebir la realidad. Los conceptos epistemológicos de la Educación Inclusiva se caracterizan por constituir estrategias de intermediación, concebidos en una especie de puente, establecen asociaciones, devaluaciones, negociaciones entre determinadas regiones del saber. Pueden también ser concebidos como sistemas de mediación epistemológica, coherente con el trabajo en las intersecciones de las disciplinas.

Otra propiedad que expresa el universo conceptual de la Educación Inclusiva es su carácter post-crítico y post-estructural, así, conceptos tales como, pluralidad, diferencia, diversidad, heterogeneidad, entre otras, se sostienen en el centro crítico de la multiplicidad. Esto nos lleva al reconocimiento que su universo conceptual se ensambla a partir de legados proporcionados por diversos movimientos críticos contemporáneos, especialmente, por discursos y políticas feministas, antirracistas, queer, etc. Interesa entonces, analizar cómo cada uno de estos discursos va tomando forma en múltiples corrientes críticas de las últimas décadas. Los conceptos a decir de Deleuze y Guattari (1990) y, contemporáneamente, por Bal (2002) pueden ser concebidos como un tratado metodológico, operan en tanto disposiciones concretas de 
configuración, son acontecimientos, esto es, actúan sobre un evento, sujetando la realidad o bien, contribuyendo a su transformación.

Los acontecimientos de acuerdo con De Mussy y Valderrama (2009), no describen exactamente aquello que ocurre, sino más bien, algo que actúa en lo que ocurre, "no es algo presente, sino algo que busca darse en lo que está presente" (De Mussy y Valderrama, 2009, p.42). Los conceptos epistemológicos son concebidos entonces como algo que actúa en un hecho, se caracterizan por poseer una fuerza conceptualizadora, creando condiciones metodológicas e interpretativas. Los conceptos se realizan en los hechos, adquieren presencia, siempre de forma provisional, se convierten así, en singularidades irreductibles. El estudio del universo conceptual de la Educación Inclusiva demanda la necesidad de disponer un examen topográfico de cada uno de ellos. Para Deleuze y Guattari (1990) ningún concepto es simple, se define a partir de componentes y se organiza a partir de ellos.

Estructuralmente, un concepto es atravesado por componentes de diversa naturaleza, incluso, afirman Deleuze y Guattari (1990), que existirán conceptos que no podrán ser albergar dentro de cada uno de estos componentes. Cada concepto circunscribe a un universo que los explica. Por todo ello, se repite la idea que el concepto es una '(re)articulación', 'repartición' e 'intersección situada'. Si los conceptos formas un todo fragmentario, su orden de producción expresa un status diseminal. El estudio de los conceptos epistemológicos de la Educación Inclusiva sugiere un pensar relacional. En este campo el problema es atravesado por lo múltiple, entre objetos, métodos, influencias, sujetos, teorías, discursos, etc. Además, de estudiar la posición que cada uno de ellos ocupa. Es necesario determinar los patrones que determinan la legibilidad epistémica de dichos conceptos de acuerdo a su base epistemológica. El análisis de los conceptos epistemológicos, permiten develar la comprensión de la inclusión y su función en el mundo social. ¿Cómo pensar conceptualmente la Educación Inclusiva, que en sí misma, es pensar la educación del siglo XXI? Los conceptos epistemológicos se convierten en 'el lugar del acontecimiento' en términos foucaultianos, ¿cuáles son las categorías que actúan en la producción de los acontecimientos? Los conceptos epistemológicos de la Educación Inclusiva pueden concebirse como un conjunto crítico, propiedad reguladora del universo pedagógico y político.

Los conceptos en este campo de producción se convierten en herramientas epistemológicas y metodológicas, definen sus posibilidades teóricas, esto exige preguntarse acerca de cuáles son los conceptos que ayudan a la Educación Inclusiva a convertirse en una ciencia post-disciplinar. Todo ello, sugiere un cambio en el orden y en la naturaleza de los mismos, es decir, si el orden cambia, cambia la naturaleza de los conceptos y también la función de los problemas (Deleuze y Guattari, 1990). Esto exige examinar las lógicas de constitución de cada concepto, comprender los ejes que delimitan su fuerza conceptualizadora y definen sus posibilidades teóricas. Es tarea del trabajo con conceptos (Canguilhem, 1975) comprender las desviaciones y los equívocos de interpretación a los que nos conducen determinados conceptos, que en su forma pudiesen encontrarse mal planteados. Epistemológicamente, un factor clave será comprender los enredos genealógicos de dispersión que se observan en el núcleo constitutivo de la epistemología de la Educación Inclusiva.

Los conceptos epistemológicos poseen la capacidad de predisponer la comprensión de un determinado objeto. Se convierten en herramientas que permiten valorar y describir críticamente a qué tipo de objetos han sido aplicados. Debido a la flexibilidad, movilidad y entropía que atraviesa al campo conceptual de la Educación Inclusiva es posible observar 
diversas prácticas de utilización de conceptos -reclaman la necesidad de develar su posición epistemológica- de forma desmedida, con cierta ligereza -propiedad del aplicacionismo epistemológico- dando paso a mecanismos de abducción y contaminación lingüística. Todo ello, demuestra la aplicación errónea de ciertos conceptos. Una tarea de tal envergadura sugiere fortalecer las herramientas analíticas y hermenéuticas propias de este campo de investigación. ¿A qué ontología del lenguaje nos conducen los conceptos epistemológicos de la inclusión?, ¿mediante qué lógicas son articulados cada uno de estos instrumentos conceptuales?

El interés en el estudio de los conceptos no se encuentra disociado de una lectura crítica sobre los diversos regímenes del saber que confluyen en este campo de producción, así como, sus mecanismos de (auto)formación y (auto) reproducción, estrategias de transformación, aceptabilidad, (des)legitimidad y dislocación, todas ellas, concebidas como herramientas de regulación epistemológicas. El universo conceptual de la Educación Inclusiva se compone de instrumentos conceptuales y herramientas lingüísticas heredadas de diversa naturaleza. La traducción en este caso, resulta altamente fértil pues inspirada en los principios de la hermenéutica diatópica y analógica, articula condiciones de negociación, transformación y re-elaboración de cada concepto, respecto de la base epistemológica que posee el enfoque aquí discutido.

Los conceptos epistemológicos de la Educación Inclusiva son propiedades no-lineales, es decir, articulan su actividad mediante un movimiento incesante a través de una multiplicidad de objetos, métodos, disciplinas y campos de conocimiento, preferentemente, cuyo funcionamiento queda determinado a través de la rearticulación de cada uno de ellos. A través de la metáfora del viaje propuesta por Bal (2002) es posible atender a las diversas formas de vinculaciones, afiliaciones, herencias -epistemológicas, lingüísticas y metodológicas-. Su potencia ofrece la capacidad de describir mediante el establecimiento de un conjunto de distinciones analíticas, las principales características del objeto de la Educación Inclusiva, y, en particular, de la inscripción y de los niveles de comprensión implicados denominados Estudios sobre Educación Inclusiva (Ocampo, 2018), mediante múltiples sistemas de relación. La superposición de conceptos - procedentes desde diversas disciplinas- constituye uno de los problemas más significativos, junto a los referidos a los de autenticidad. Con el objeto de identificar los ejes que sustentan las problemáticas interpretativas, la abducción y la contaminación lingüística, así como, los ejes que participan de la superposición de sus conceptos, observo la necesidad de disponer de un trabajo analítico que dé cuenta de las diferencias entre cada uno de ellos y de la multiplicidad de marcos epistemológicos, metodológicos y políticos que participan en su configuración.

El estudio de los conceptos se convierte en una pieza crucial en los estudios epistemológicos, éstos, constituyen en sí mismos, problemas teóricos y metodológicos. Asimismo, proporcionan un grado significativo de legibilidad a un determinado campo de conocimiento especialmente, a terrenos emergentes, como es el aquí discutido-, fijan sus normas de funcionamiento y cientificidad, además de proporcionar condiciones de aplicabilidad a un determinado objeto. Para lo cual, será necesario que sus conceptos se encuentren claramente definidos. Una de sus funciones consiste en la capacidad de enfocar el interés (Bal, 2002). ¿Cuál es el papel que desempeñan los conceptos en la actividad científica de la Educación Inclusiva? Atender a esta interrogante coincide con Bal (2002), respecto de los siguientes elementos: a) la redefinición de categorías y significados, tanto en el campo fenomenológico como en el social, b) los conceptos poseen la capacidad de organizar un grupo de fenómenos, c) determinan 
preguntas relevantes y c) significados que pueden ser atribuidos al observador. El universo conceptual de la Educación Inclusiva observa en su versión falsificada e hibridizada que deviene en un campo difuso, en la aplicación ligera e injustificada de ciertos conceptos, que a su vez, carecen de estatus metodológico, es decir, capacidad de explicar o describir analíticamente un determinado fenómeno y sus ejes de especificidad. Su función queda reducida según Bal (2002) a una forma simple de nombrar.

La imagen que se expone a continuación, tiene por objeto sintetizar las principales características epistemológicas, metodológicas y conceptuales implicadas en la comprensión de la Educación Inclusiva.

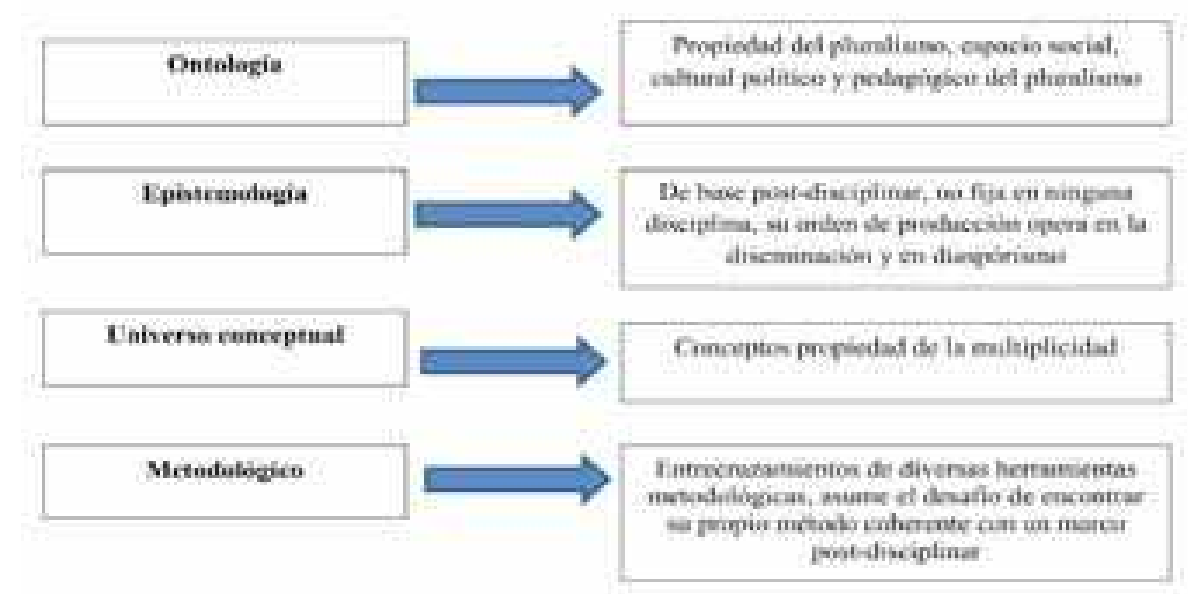

Figura 2. Síntesis de niveles de comprensión analitico-metodologicas implicadas en el estudio de la Educación Inclusiva. Fuente: Elaboración propia.

\section{6.-CONCLUSIONES}

La comprensión epistemológica de la Educación Inclusiva no sólo demanda el estudio de sus condiciones de producción, sino más bien, cómo determinados sistemas de razonamientos crean particulares regímenes de verdad, los cuáles, pasan inadvertidos al interior de su campo intelectual, de la formación de los educadores, en prácticas de investigación y en las políticas públicas. Concebir la Educación Inclusiva como una propiedad sustantiva de la praxis educativa, sugiere el reconocimiento que, el respeto a las diferencias, el pluralismo, el reconocimiento del Otro, la ampliación de oportunidades, la construcción de justicia son propiedades centrales de la tarea educativa y no de una práctica especializada -como erróneamente se ha creído. Sin duda, develar las causas de dicha distorsión, observo, constituye una empresa fértil-. Por tanto, ubicar su objeto en la educación permite reconocer la esencia que progresivamente ha sido extraviada en el sentido más intrínseco de la misma.

En los últimos años el concepto de inclusión ha designado un terreno fundamentalmente disputado por una multiplicidad de disciplinas académicas, discursos, concepciones políticas y corrientes críticas más prominentes de nuestra época. ¿Cómo llega a ocupar esta posición?, intentar ofrecer una respuesta a una interrogante de estas características, sugiere emprender una crítica genealógica sobre el conjunto de condiciones sociales, institucionales e intelectuales que propician su objeto de conocimiento. Uno de los propósitos en este marco, consistirá en cuestionar los diversos tipos de relaciones a través de la pluralidad de elementos que dan vida al presente campo de estudio. Coincidiendo 
con Knorr (2005) y Mowitt (1992), es menester profundizar en el estudio de la lógica cultural disciplinaria que permea su funcionamiento y campo de acción. Sugiere además, develar los problemas post-disciplinarios que este enfrente en su desarrollo.

La comprensión de la Educación Inclusiva como praxis social, cultural y política sugiere reconocer que los conceptos -requieren develar su estatus metodológico, con el objeto de convertirlos en categorías de análisis- de inclusión, totalidad concebida como singularidades múltiples, lo común, la diferencia, etc., se convierten en ejes relacionales subyacentes en lo más profundo del campo epistemológico de la Educación Inclusiva, intentando develar sus significados a través de diversas posturas críticas. Una posible respuesta, puede encontrarse en el develamiento de los marcos políticos y teóricos en los que se inscribe su fenómeno, cuyo examen sugiere atender heurísticamente la inscripción y resignificación del campo cultural, social y político del derecho 'en' la educación, preferentemente. Por todo ello, es menester concebir el sentido y alcance de la Educación Inclusiva como un mecanismo de transformación de todos los campos de la Ciencia Educativa. Su fuerza performativa, moviliza un discurso de alteración de los lenguajes, vocabularios, marcos y formas de pensar y experimentar la educación para la multiplicidad de diferencias. Orienta su actividad hacia la creación de conceptos, herramientas metodológicas y saberes que permitan leer críticamente el presente. Se convierte así, en una expresión de la historicidad del presente.

\section{REFERENCIAS}

Agamben, G. (2006). ¿Qué es un dispositivo? Recuperado el 12 de febrero de 2018: http://ayp.unia.es/r08/lMG/pdf/ agamben-dispositivo.pdf

Arditi, B. (2007). Politics on the Edges of Liberalism: Difference, Populism,
Revolution, Agitation. Edinburgh: Edinburgh University Press.

Bal, M. (2002). "Conceptos viajeros en las humanidades". Estudios visuales. Ensayo, teoría y crítica de la cultura visual y el arte contemporáneo, 3, 2877.

Bal, M. (2003). "From Cultural Studies to Cultural Analysis: A Controlled Reflection on the Formation of Method", en: Bowman, P. (ed.). Interrogating Cultural Studies Theory, Politics and Practice. London: Pluto Press. 30-40.

Bal, M. (2016). Tiempos trastornados. Análisis, historias y políticas de la mirada. Madrid: Akal.

Bowman, P. (2008). "Alterdisciplinarity". Culture, Theory and Critique, 49:1, 93-110.

Canguilhem, G. (1975). La formation du concept de réflexe aux XVIle et XVIIle siècles. París: PUF.

Deleuze, G. (1990). “¿Qué es un dispositivo?”, en: VV.AA. Michel Foucault Filósofo. Barcelona: Gedisa.

Deleuze, G. \& Guattari, F. (1990). ¿Qué es la filosofía? Barcelona: Anagrama.

Deleuze, G. \& Guattari, F. (1970). Mil mesetas. Capitalismo y esquizofrenia. Valencia: Pre-Textos.

De Mussy, L. \& Valderrama, M. (2009). Historiografía postmoderna: conceptos, figuras y manifiestos. Santiago: RIL/ Universidad Finnis Terrae.

Derrida, J. (1997). La diseminación. Madrid: Fundamentos.

Derrida, J. (2003). 'I Have a Taste for the Secret'; en: Derrida, J. y 
Ferraris, M. (Comp.). A Taste for the Secret. Cambridge: Polity Press, 1-18.

Fornet-Betancourt, R. (1994). Filosofía Intercultural. México: UPM.

Foucault, M. (1973). La verdad y las formas jurídicas. Barcelona: Gedisa.

Knorr, K. (2005). La fabricación del conocimiento. Un ensayo sobre el carácter constructivista y contextual de la ciencia. Buenos Aires: Ed. Univ. Nacional de Quilmes.

Mowitt, J. (1992). Text: The Genealogy of an Antidisciplinary Object. Durham and London: Duke.

Ocampo, A. (2016). "Gramática de la Educación Inclusiva. Ejes críticos para cartografiar sus condiciones de producción y funcionamiento epistémico", en: Ocampo, A. (Coord.). Ideología, Invisibilidad y Dominación. Los imaginarios constitutivos de la discapacidad en Latinoamérica. Ediciones CELEI: Santiago, 73-159.

Ocampo, A. (2018). La formación del profesorado y la comprensión epistemológica de la Educación Inclusiva: tensiones, permeabilidades y contingencias. Santiago: Ediciones CELEI.

Pratt, M.L. (1992). Imperial Eyes: writing and transculturation. Routledge: London.

Sousa, B. (2009).Una epistemología del sur: la reinvención del conocimiento y la emancipación social. México: Siglo XXI/ Clacso.

Spivak, G. (2008). "Estudios de la Subalternidad. Deconstruyendo la historiografía", en: Mezzadra, S., Spivak, G., Tapadle, Ch., Sota, E., Hall, S. Chakrabarty, D., Mbembe, A., Young, R., Puwar, N. y Róala, F. Estudios Postcoloniales. Ensayos fundamentales. Madrid: Traficante de Sueños, pp. 33-68.

Vandenberghe, F. (2004). Las condiciones de posibilidad de conocimiento del objeto y del objeto de conocimiento en sociología. Revue du mauss, vol. 24, núm. 2, pp. 315-329. 